\title{
Presentation of COVID-19 Disease and the Impact of Patient's Comorbidities on Its Hospital Outcome: An Observational Study in a COVID-19 Dedicated Hospital
}

\author{
M. M. Bodiuzzaman and M. I. Hossain
}

\section{ABSTRACT}

Background: Coronavirus disease (COVID-19) caused by severe acute respiratory syndrome coronavirus 2 (SARS-CoV-2) is a global pandemic that initially started in Wuhan, China, and spread exponentially across the globe infecting human being irrespective of age, sex and ethnicity. Given the nature of this virus, there is much still to be learned. People with COVID19 have had a wide range of symptoms reported - ranging from mild symptoms (e.g., common cold) to more severe diseases such as bronchitis, pneumonia, severe acute respiratory distress syndrome (ARDS), multiorgan failure, and even death. Comorbidity is an important factor in COVID-19 pandemic outcome often leading to rapid and severe progression of the disease process, even death.

Objective: This study was carried out to see the socio-demographic characteristics and presenting features of COVID-19 disease as well as to assess impact of comorbidities on its hospital outcome.

Methods: This observational study was carried out in the COVID-19 dedicated hospital of Faridpur from April 2020 to September 2020 for a period of 06 month. All clinically suspected patients confirmed by RT PCR were included as cases. Data were collected by detailed history from patients then those were checked, verified for consistency and edited for result. After editing and coding, the coded data were analyzed by using the SPSS $\backslash P C$ software package.

Results: A total of 627 patients were included in the study of which 552 were treated in COVID ward and 75 patients were treated in ICU. Among COVID ward admitted (552) patients $354(64.13 \%)$ were male and 198 $(35.86 \%)$ were female with a male to female ratio of 1:0.56; young adult patients (19 to 50 years) were more affected and admitted $(62.86 \%)$ and people living in urban area were more affected $(52.71 \%)$ than rural area $(47.28 \%)$. Fever, cough and shortness of breath $(63.04 \%, 45.47 \%$ and 42.39\% respectively) were predominant symptoms. Regarding comorbidities, $\mathbf{4 4 . 2 0 \%}$ patients have one or more comorbidities whereas $55.79 \%$ patients have no comorbidity. Hypertension $(\mathbf{1 7 . 5 7 \%})$ was the predominant comorbid condition followed by Diabetes $(15.94 \%)$, ischemic heart disease $(05.61 \%)$, COAD $(05.61 \%)$, CKD (2.3\%), Stroke $(1.44 \%)$, Heart failure $(0.54 \%)$ and Cancer $(0.36 \%)$. A total of 75 patients needed ICU support; most of them were elderly patients (64 out of 75). Regarding hospital outcome, 96.74\% (534 out of 552) COVID ward patients and $45.34 \%$ (34 out of 75 ) of ICU admitted patients discharged uneventfully whereas $03.2 \%$ COVID ward patients and $54.66 \%$ of ICU admitted patients expired. Deaths were more in elderly patients $(n=43 ; 72.88 \%)$. Common comorbidities found among the patients who expired were Hypertension, Diabetes and Ischemic heart diseases $(42.37 \%, 37.28 \%$ and $16.94 \%$ respectively).

Conclusion: The predominant number of patients presented with fever, cough and shortness of breath in our setting. The percentage of COVID-19 hospitalizations resulting in death remains high among elderly patients and those with one or more comorbid conditions. Therefore, elderly patients and those with comorbidities should take all necessary precautions to avoid getting infected with SARS CoV-2.

Keywords: Coronavirus, COVID-19, SARS-CoV-2, Clinical features, Comorbidity.
Submitted : March 27, 2021

Published : April 17, 2021

ISSN: $2593-8339$

DOI: $10.24018 /$ ejmed.2021.3.2.788

M. M. Bodiuzzaman *

Department of Medicine, Faridpur Medical College, Faridpur, Bangladesh. (e-mail: drbodiuzzaman72@gmail.com) M. I. Hossain

Department of Medicine, Faridpur Medical College, Faridpur, Bangladesh.

*Corresponding Author 


\section{INTRODUCTION}

An outbreak of novel coronavirus (SARS-CoV-2) emerged in December 2019 in Wuhan of China has led to a global pandemic, affecting around 250 countries across the globe. The virus, named severe acute respiratory syndrome coronavirus 2 (SARS-CoV-2), causes a clinical syndrome termed coronavirus disease 2019 (COVID-19). SARS-CoV2 is predominantly spread by respiratory droplets and by contaminated fomites as well as by aerosols in certain circumstances [1].

Several studies have estimated the incubation period of COVID-19. Based on the experience in China, the typical incubation period of COVID-19 infection has been estimated to be a median of 5.1 days. The reported mean incubation period for COVID-19 varied from 4 days to 10.9 days. Incubation period distributions of different age groups are significantly different. Of symptomatic cases in any age group, about $95 \%$ will show symptoms within 14 days. This supports the currently practiced length of quarantine in many countries [2], [3].

The virus infects humans in all age groups, of all ethnicities, both males and females. Older age, male sex, obesity, hypertension, diabetes, cardiovascular disease and chronic lung disease, Chronic kidney disease (CKD) have shown worse prognosis and are risk factors for COVID-19 mortality [4]-[6].

Hypertension and diabetic patients have increased morbidity and mortality rates and have been linked to more hospitalization and intensive care unit (ICU) admissions [4], [5]. Pre-existing COPD is likely to worsen the progression and prognosis of COVID-19 [7]. Elderly population is more susceptible to this illness and is more likely to be admitted to the ICU with a higher mortality rate. The age-related changes in the geriatric population may be due to the changes in lung anatomy and muscle atrophy which results in changes in physiologic function, reduction of lung reserve, reduction of airway clearance, and reduction of the defense barrier function [8].

COVID-19 infection has a broad spectrum of severity ranging from an asymptomatic form to a severe acute respiratory syndrome that requires mechanical ventilation. Among symptomatic patients, about $80 \%$ showed a mild clinical course characterized by a dry cough, sore throat, lowgrade fever, or malaise; in $20 \%$ of cases, the general condition worsened in about seven days from the beginning of the symptoms, culminating in respiratory failure [9].

As the novel coronavirus continues to evolve, there are still many limitations to our knowledge of who exactly this virus would impact critically.

\section{Methods AND MATERIALS}

This observational study was carried out in the COVID-19 dedicated hospital of Faridpur (situated in Faridpur Medical College Hospital, Faridpur) from April 2020 to September 2020 for a period of 06 month. All clinically suspected patients confirmed by RT PCR were included as cases and those who were not confirmed, excluded from this study. Cases were selected irrespective of age and sex on a random basis. Patients admitted in corona ward were selected for symptom analysis, comorbidities and hospital outcome whereas patients admitted in ICU were excluded from symptom analysis. Data were collected by detailed history from patients, or their relatives followed by thorough physical examination as well as diagnostic evaluation; then those were checked, verified for consistency and edited for result. After editing and coding, the coded data were analyzed by using the SPSS $\backslash$ C software package.

\section{RESULT}

A total of 627 patients were included in the study of which 552 were treated in COVID ward and 75 patients were treated in ICU. Among COVID ward admitted (552) patients 354 $(64.13 \%)$ were male and $198(35.86 \%)$ were female with a male to female ratio of 1:0.56; young adult patients (19 to 50 years) were more affected and admitted $(62.86 \%)$ and people living in urban area were more affected $(52.71 \%)$ than rural area $(47.28 \%)$ (Table I).

TABLE I: Distribution OF PATIENTS ACCORDING TO AGE, SEX AND

\begin{tabular}{|c|c|c|}
\hline \multicolumn{2}{|c|}{$\begin{array}{l}\text { RES } \\
\text { Demographics }\end{array}$} & \multirow{2}{*}{$\begin{array}{c}\text { Frequency }(\%) \\
24(4.3)\end{array}$} \\
\hline \multirow{3}{*}{ Age group } & $<19$ years & \\
\hline & $19-50$ years & 347 (62.86) \\
\hline & $>50$ years & $181(32.78)$ \\
\hline \multirow{2}{*}{ Sex } & Male & $354(64.13)$ \\
\hline & Female & $198(35.86)$ \\
\hline \multirow{2}{*}{ Residence } & Urban & $291(52.71)$ \\
\hline & Rural & $261(47.28)$ \\
\hline
\end{tabular}

Fever, cough and shortness of breath $(63.04 \%, 45.47 \%$ and $42.39 \%$ respectively) were predominant symptoms followed by asymptomatic and other symptoms like sore throat, headache, generalized body ache and other non -respiratory problems (Table II).

TABLE II: DISTRIBUTION OF PATIENTS ACCORDING TO PRESENTATION

\begin{tabular}{cc} 
& $(\mathrm{N}=552)$ \\
\hline Symptoms & Frequency $(\%)$ \\
\hline Fever & $348(63.04)$ \\
Cough & $251(45.47)$ \\
SOB & $234(42.39)$ \\
Sore throat & $24(4.34)$ \\
Headache & $16(2.8)$ \\
Generalized bodyache & $13(2.3)$ \\
Asymptomatic & $46(8.33)$ \\
Others & $14(2.3)$ \\
\hline
\end{tabular}

Regarding comorbidities, $44.20 \%$ patients have one or more comorbidities whereas $55.79 \%$ patients have no comorbidity. Hypertension $(17.57 \%)$ was the predominant comorbid condition followed by diabetes $(15.94 \%)$, ischemic heart disease $(05.61 \%)$, COAD $(05.61 \%)$, CKD $(2.3 \%)$, Stroke $(1.44 \%)$, Heart failure $(0.54 \%)$ and Cancer $(0.36 \%)$ (Table III).

TABLE III: DISTRIBUTION OF PATIENTS ACCORDING TO COMORBIDITIES

\begin{tabular}{cc}
\multicolumn{2}{c}{$\begin{array}{c}\text { TABLE III: DISTRIBUTION OF PATIENTS ACCORDING TO COMORBIDITIES } \\
(\mathrm{N}=552)\end{array}$} \\
\hline Comorbidities & Frequency $(\%)$ \\
\hline Hypertension (HTN) & $97(17.57)$ \\
Diabetes Mellitus (DM) & $88(15.94)$ \\
Ischemic Heart Disease (IHD) & $31(5.61)$ \\
Heart failure (HF) & $03(0.54)$ \\
Chronic Obstructive Airway Diseases & $31(5.61)$ \\
(COAD) & $13(2.3)$ \\
Chronic kidney disease (CKD) & $08(1.44)$ \\
Cerebrovascular disease (CVD) & $02(0.36)$ \\
Cancer &
\end{tabular}


Regarding hospital outcome, 96.8\% COVID ward patients and $45.34 \%$ of ICU admitted patients discharged uneventfully whereas $03.26 \%$ COVID ward patients and $54.66 \%$ of ICU admitted patients expired. Deaths were more in elderly patients $(n=43 ; 72.88 \%)$. Common comorbidities found among the patients who expired were Hypertension, Diabetes and Ischemic heart diseases $(42.37 \%, 37.28 \%$ and $16.94 \%$ respectively). A total of 75 patients needed ICU support that was $11.96 \%$ of total cases; most of them were elderly patients (64 out of 75 i.e., $85.33 \%$ ). Out of total mortality, death rate was much higher in ICU than in COVID ward $(69.49 \%$ vs $30.50 \%)$. The male to female ratio of ICU death was 3.2:1. The mortality rate in ICU was higher in those with one or more comorbid conditions; the predominant comorbidities were hypertension $(42.37 \%)$ and diabetes $(37.28 \%)$ followed by IHD, COAD, CKD and CVD. No comorbidity was found in $27.11 \%$ of ICU death (Table IV, V, VI).

TABLE IV: DISTRIBUTION OF PATIENTS ACCORDING TO ICU TREATMENT AND OUTCOME

\begin{tabular}{cccc}
\hline \multicolumn{3}{c}{ AND OUTCOME } \\
\hline \multicolumn{2}{c}{$\begin{array}{c}\text { Age Group of ICU Treated } \\
\text { Patients } \\
(\mathrm{n}=75,11.96 \%)\end{array}$} & $>51$ year & $11(14.66 \%)$ \\
\cline { 3 - 4 } & Recovery & COVID Ward & $64(85.33 \%)$ \\
\hline (n=568) & ICU & $534(96.74 \%)$ \\
Outcome & Death in Total & COVID Ward & $18(3.26 \%)$ \\
$(\mathrm{n}=627)$ & cases (n=627) & ICU & $41(54.66 \%)$ \\
& Comparison of & COVID Ward & $18(30.50 \%)$ \\
& death $(\mathrm{n}=59)$ & ICU & $41(69.49 \%)$ \\
\hline
\end{tabular}

TABLE V: Distribution of MORTALity aCCORDing to Age, SEX AND COMORBIDITIES $(\mathrm{N}=59)$

\begin{tabular}{ccc}
\hline & & Frequency $(\%)$ \\
\hline Age group & <19 years & $02(3.38)$ \\
& 19-50 years & $14(23.72)$ \\
Sex & $>50$ years & $43(72.88)$ \\
& Male & $45(76.27)$ \\
Comorbidities & Female & $14(23.72)$ \\
& Comorbidity present & $43(72.88)$ \\
& Comorbidity absent & $16(27.12)$ \\
\hline
\end{tabular}

TABLE VI: DISTRIBUTION OF MORTALITY ACCORDING TO PATTERN OF

\begin{tabular}{ccc}
\multicolumn{3}{c}{ COMORBIDITIES (N=59) } \\
\hline $\begin{array}{c}\text { Co morbidity in dead } \\
\text { patients }\end{array}$ & Comorbidities & Frequency $(\%)$ \\
\hline & HTN & $25(42.37)$ \\
& DM & $22(37.28)$ \\
Present $(n=43)$ & IHD & $10(16.94)$ \\
& COAD & $07(11.86)$ \\
& CKD & $04(6.77)$ \\
Absent $(n=16)$ & CVD & $04(6.77)$ \\
& Others & $04(6.77)$ \\
\hline
\end{tabular}

\section{DISCUSSION}

In this study a total of 627 patients were included (552 from Covid ward and 75 from ICU) and demographic, clinical presentation as well as pertinent data regarding impact of comorbidities on COVID-19 disease outcome were collected.

In our study, adult patients especially the economically productive age group i.e., 19-50 were mostly affected $(62.86 \%)$ followed by elderly population $(32.78 \%)$. The percentage of under 19 with confirmed COVID-19 cases is far lower $(4.3 \%)$ than the standard population percentage. These findings were closely related to a review done by Dominic Cortis, where three studies were included. Two studies were from China by Zhang and Guan et al. and another one from South Korea by Korea Centers for Disease Control and Prevention. Those studies showed that the percentage of youths with confirmed COVID-19 cases is far lower than the standard population percentage. The proportion of COVID-19 confirmed cases for youths (age group 0-14 year:) is lower in China $(1.55 \%, 0.89 \%)$ than South Korea $(4.04 \%)$. The predominant population affected in all three studies were 15-64year groups (76.93\%, 83.98\% in China and $78.60 \%$ in S. Korea) followed by elderly population $(21.53 \%, 15.13 \%$ in China and $17.36 \%$ in $\mathrm{S}$. Korea) [10]. A study conducted in China showed the age distribution for all patients where $61.5 \%$ were aged $<60$ years and the other cases were aged $\geq 60$ years; this is consistent with our studies $(67.16 \%$ in below 50 group vs $32.78 \%$ in above 50 group) [11]. A study conducted in India showed that 21-50 age group, contributes to the maximum proportion $(60 \%)$ of the total cases followed by those below 20 years of age constituting nearly $13 \%$ of the cases [12]. There is a deviation of this study to ours as well as to China and South Korean studies in respect of younger peoples' Covid-19 positivity.

Regarding sex distribution, in a study that included a total of 5700 patients admitted into 12 different hospitals of USA found $39.7 \%$ female and $60.3 \%$ male as Covid 19 positive [13]. In another study conducted in India males contribute to $66 \%$ of the total positive cases [12]. In a study in India, it is observed that women are half as likely to be infected by COVID-19 as men [14]. These above-mentioned results almost match with our study result (male $64.13 \%$ and female $35.86 \%$ ).

In our study we found, urban population affected more $(52.71 \%)$ than rural population $(47.28 \%)$. There may be some explanations linking urban areas and coronavirus, emphasizing densities; connectivity; crowded living conditions; and exposed occupations.

Regarding presentation, in this study, most of the patients presented with fever $(63.04 \%)$, cough $(45.47 \%)$ and shortness of breath $(42.39 \%)$. The less predominant symptoms were sore throat $(4.34 \%)$, headache $(2.8 \%)$ followed by with chest pain, abdominal pain, diarrhea, vomiting, bleeding manifestation and psychosis in a minor of patients. There were $8.33 \%$ of asymptomatic patients. In a meta-analysis that included seven articles published from 24th Jan to 16th March 2020 revealed that fever was the predominant symptom $(88.8 \%)$ followed by dry Cough $(68 \%)$ fatigue (33\%), productive cough $(28.5 \%)$, muscle pains $(14.4 \%)$, diarrhea $(4.4 \%)$, nausea or vomiting $(4.1 \%)$, rhinorrhea (3.2\%), chest and abdominal pain $(0.15 \%)$ [15]. Similarly, a study in a hospital of Wuhan, China found fever (98\%), cough $(76 \%)$, dyspnoea $(55 \%)$, myalgia or fatigue $(44 \%)$, sputum production $(28 \%)$, headache $(8 \%)$, haemoptysis (5\%), and diarrhoea (3\%) as common symptoms [16]. In another meta-analysis, found similar result, where most prevalent clinical symptom was fever $(91.3 \%)$, followed by cough (67.7\%), fatigue (51.0\%) and dyspnea (30.4\%) [17]. These above-mentioned studies closely matched with the result of our study.

Considering comorbidity, $44.24 \%$ patients had one or more comorbidities and $55.79 \%$ presented in isolation. Common comorbid conditions found were as follows: HTN (17.57\%), 
DM (15.94\%), IHD (5.61\%), COAD $(5.61 \%)$ followed by CKD $(2.3 \%)$, CVD (1.44\%), Heart failure $(0.54 \%)$ and Cancer $(0.36 \%)$.

In a meta-analysis, as mentioned above revealed hypertension $(15.8 \%)$ as the most common comorbidity followed by other cardiovascular and cerebrovascular conditions $(11.7 \%)$, endocrine disorder primarily diabetes (9.4\%), co-existing infection like HIV and Hepatitis B $(1.5 \%)$, malignancy $(1.5 \%)$, respiratory system disorder, e.g., COPD and others $(1.4 \%)$, renal disorders $(0.8 \%)$ and immunodeficiency states $(0.01 \%)$ [15]. Almost similar results were found in another retrospective, multicenter cohort study, where $48 \%$ patients had comorbidities, with hypertension being the most common $(30 \%)$, followed by diabetes $(19 \%)$ and coronary heart disease $(8 \%)$ [18]. In a population-based surveillance for laboratory-confirmed COVID-19-associated hospitalizations in the United States, among 1,482 patients, $12 \%$ adult patients had one or more underlying conditions; the most common were hypertension $(49.7 \%)$, obesity (48.3\%), chronic lung disease $(34.6 \%)$, diabetes mellitus $(28.3 \%)$, and cardiovascular disease $(27.8 \%)$ [19].

The results of first two studies closely resemble with our study (Hypertension, Coronary artery disease and Diabetes as predominant comorbidities), but the third one revealed obesity as an important comorbidity which was not included in our study.

In our study, $96.8 \%$ covid ward patients and $45.34 \%$ of ICU admitted patients discharged uneventfully whereas $03.2 \%$ covid ward patients and $54.66 \%$ of ICU admitted patients expired. These matches with the following two studies.

Approximately $10 \%$ of the global population may have been infected by October 2020, with an estimated overall IFR of $0.15 \%$ to $0.2 \%$ ( $0.03 \%$ to $0.04 \%$ in those $<70$ years of age) [20]. In another study, roughly $80 \%$ of COVID-19-positive cases result in full recovery from the illness without any hospitalizations or interventions [5].

In our study, death was more in elderly patients $(n=43$; $72.88 \%$ ). A total of 75 patients needed ICU support that was $11.96 \%$ of total cases; most of them were elderly patients (64 out of 75 i.e., $85.33 \%$ ). Out of total mortality, death rate was much higher in ICU than in COVID ward $(69.49 \%$ vs $30.50 \%)$. These results are coherent with the studies done in other centers.

COVID-19 can cause severe disease leading to hospitalization in ICU and potentially death, especially in the elderly with comorbidities. According to the CDC, 8 out of 10 deaths reported in the USA occurred in adults 65 years old and above.5 According to a report by CDC, data from China have indicated that older adults, particularly those with serious underlying health conditions, are at higher risk for severe COVID-19-associated illness and death than are younger persons. In the same report, COVID-19 cases in the United States, Overall, $31 \%$ of cases, $45 \%$ of hospitalizations, $53 \%$ of ICU admissions, and $80 \%$ of deaths associated with COVID-19 were among adults aged $\geq 65$ years with the highest percentage of severe outcomes among persons aged $\geq 85$ years [21]. People $<65$ years of age have a very small risk of death even in pandemic epicenters, and deaths in people $<65$ years of age without any underlying conditions is rare [22]. In our study, the mortality rate in ICU was higher in those with one or more comorbid conditions; the predominant comorbidities were hypertension $(42.37 \%)$ and diabetes (37.28\%) followed by IHD, COAD, CKD and CVD. No comorbidity was found in $27.11 \%$ of death. In Italy only $12 \%$ of death certificates reported direct causality from COVID19 , while $88 \%$ of patients who died had at least one comorbidity [23], [24]. In New York state, just over $86 \%$ of reported COVID-19 deaths involved at least one comorbidity, according to the state's department of health. The leading comorbidity, seen in $55.4 \%$ of all deaths, was hypertension. the rest of the 10 most common comorbidities in COVID-19 fatalities were diabetes $(37.3 \%)$, hyperlipidemia $(18.5 \%)$, coronary artery disease $(12.4 \%)$, renal disease $(11.0 \%)$, dementia $(9.1 \%)$, chronic obstructive pulmonary disease $(8.3 \%)$, cancer $(8.1 \%)$, atrial fibrillation $(7.1 \%)$, and heart failure $(7.1 \%)$ [25].

In our study, HTN, DM and COAD patients shows more mortality rates than New York City study. Hyperlipidemia and Dementia were not included in ours.

\section{LIMITATION}

The first limitation of this study is the relatively small number of patients included vast majority of the patients were either treated outside the hospital or were asymptomatic. Secondly, all possible comorbidities were not included in the study.

\section{CONCLUSION}

The coronavirus disease 2019 (COVID-19) has rapidly spread to become a worldwide emergency. Most of the patients presented with fever, cough and respiratory distress in our setting. The elderly patients and those with one or more comorbid conditions reflected poor outcomes.

The study highlights the importance of early identification of patients at risk of progression for optimized utilization of medical resource. There are still many facts to be elucidated, reflecting our uncertainty regarding this disease.

\section{ACKNOWLEDGEMENT}

Authors of the study are thankful to all healthcare providers of COVID-19 dedicated hospital of Faridpur for their cooperation.

\section{REFERENCES}

[1] Brewster DJ, Chrimes N, Do TB, Fraser K, Groombridge CJ, Higgs A, et.al. Consensus statement: Safe Airway Society principles of airway management and tracheal intubation specific to the COVID-19 adult patient group. Med J Aust. 2020 Jun; 212(10): 472-81. doi:10.5694/mja2.50598. Epub 2020 May.

[2] Lauer SA, Grantz KH, Bi Q,Jones FK, Zheng Q, Meredith HR, et al. The incubation period of coronavirus disease 2019 (COVID-19) from publicly reported confirmed cases: estimation and application. Ann Intern Med.2020 Mar ;172: 577- 82, doi:10.7326/M20-0504.

[3] Dai J, Yang L, Zhao J. Probable Longer Incubation Period for Elderly COVID-19 Cases: Analysis of 180 Contact Tracing Data in Hubei Province, China. Risk Manag Healthc Policy. 2020; 13: 1111-17.doi: 10.2147/RMHP.S257907.

[4] Singh AK, Gupta R, Ghosh A, Misra A. Diabetes in COVID-19: prevalence, pathophysiology, prognosis, and practical considerations. 
Diabetes Metab Syndr. 2020;14(4):303-10.doi: 10.1016/j.dsx.2020.04.004.

[5] Sanyaolu A, Okorie C, Marinkovic A, Patidar R, Younis K, Desai P, et al. Comorbidity and its Impact on Patients with COVID-19.SN Compr Clin Med. 2020 Jun 25: 1-8.doi: 10.1007/s42399-020-00363-4.

[6] Gansevoort RT, Hilbrands LB.CKD is a key risk factor for COVID-19 mortality. Nat Rev Nephrol. 2020 Aug. https://doi.org/10.1038/s41581-020-00349-4.

[7] Zhao Q, Meng M, Kumar R, Wu Y, Huang J,Lian N,et al.The impact of COPD and smoking history on the severity of COVID-19: a systemic review and meta-analysis. J Med Virol. 2020 May;92(10)1-7.doi: 10.1002/jmv.25889.

[8] Liu K, Chen Y, Lin R, Han K. Clinical features of COVID-19 in elderly patients: a comparison with young and middle-aged patients. J Infect. 2020 Mar; 80(06): E14-E18. DOI: https://doi.org/10.1016/j.jinf. 2020.03.005.

[9] Macera M, Angelis GD, Sagnelli C, Coppola N, Vanvitelli COVID-19 Group. Clinical Presentation of COVID-19: Case Series and Review of the Literature. Int J Environ Res Public Health. 2020 Jul; 17(14): 5062.doi: 10.3390/ijerph17145062.

[10] Cortis D. On Determining the Age Distribution of COVID-19 Pandemic. Front Public Health. 2020 May; 8: 202. doi:10.3389/fpubh. 2020.00202.

[11] Liu Y, Mao B, Liang S, Yang J-W, Lu H-W, Chai Y-H, et al. Association between age and clinical characteristics and outcomes of COVID-19. Eur Respir J.2020 May;55(5):2001112. doi:10.1183/13993003.01112-2020.

[12] Kulkarni SV, Chauhan H. COVID-19 in Different Age Groups of Children: Initial Impression from Integrated Disease Surveillance Programme (IDSP) under National Centre for Disease Control (NCDC). Indian J Pediatr. 2020 Jul; 87:674-5. Doi:10.1007 /s12098020-03457y.

[13] Richardson S, Hirsch JS, Narasimhan M, Crawford JM, McGinn T, Davidson KW, et al. Presenting Characteristics, Comorbidities, and Outcomes Among 5700 Patients Hospitalized With COVID-19 in the New York City Area. JAMA.2020;323(20):2052-59. doi:10.1001/jama.2020.6775.

[14] Gupta S. The age and sex distribution of COVID-19 cases and fatalities in India. medRxiv 187953 [preprint]. 2020 [Jul 16] doi:10.1101/2020.07.14.20153957.

[15] Paudel SS. A meta-analysis of 2019 novel corona virus patient clinical characteristics and comorbidities. Research Square 148580 [preprint].2020 [Apr 08]. Available from doi: 10.21203/rs.3.rs21831/v1.

[16] Huang C, Wang Y, Li X, Ren L, Zhao J, Hu Y, et al. Clinical features of patients infected with 2019 novel coronavirus in Wuhan, China Lancet.2020 Feb 15;395 (10223):497-506. doi:10.1016/S01406736(20)30183-5.

[17] Yang J, Zheng Y, Gou X, Pu K, Chen Z, Guo Q, et al. Prevalence of comorbidities and its effects in patients infected with SARS-CoV-2: a systematic review and meta-analysis. Int J Infect Dis.2020 May;94:915.doi:10.1016/j.ijid.2020.03.01. Epub 2020 Mar 12.

[18] Zhou F, Yu T, Du R, Fan G, Liu Y, Liu Z, et al. Clinical course and risk factors for mortality of adult inpatients with COVID-19 in Wuhan, China: a retrospective cohort study. Lancet.2020 Mar 28 ;395 (10229): 1054-62. doi:10.1016/S0140-6736(20)30566-3.

[19] Garg S, Kim L, Whitaker M, O’Halloran A, Cummings C, Holstein R, et al. Hospitalization Rates and Characteristics of Patients Hospitalized with Laboratory-Confirmed Coronavirus Disease 2019- COVID-NET, 14 States. MMWR. 2020 April 17;69(15):458-64.

[20] Ioannidis JPA. Global perspective of COVID-19 epidemiology for a full-cycle pandemic. Eur J Clin Invest. 2020;00: e13423. doi: 10.1111/eci.13423

[21] Bialek S, Boundy E, Bowen V, Chow N, Cohn A, Dowling N et al. Severe Outcomes Among Patients with Coronavirus Disease 2019 (COVID-19) -United States, February 12-March 16, 2020. MMWR: Morb Mortal Wkly Rep. 2020 Mar 27;69(12): 343-46. doi: 10.15585/mmwr .mm6912e2.

[22] Loannidis JPA, Axfors C, Contopoulos-Ioannidis DG. Population-level COVID-19 mortality risk for non-elderly individuals overall and for non-elderly individuals without underlying diseases in pandemic epicenters. Environ Res. 2020 Sep; 188:109890. doi doi:10.1101/2020.04.05.20054361.

[23] Oke J, Heneghan C.Global COVID-19 case fatality rates. 2020 [internet publication]. Centre for Evidence-Based Medicine;2020.

[24] Onder G, Rezza G, Brusaferro S. Case-fatality rate and characteristics of patients dying in relation to COVID-19 in Italy. JAMA. 2020;323(18):1775-6. doi:10.1001/jama.2020.4683.

[25] Franki R. Comorbidities the rule in New York's COVID-19 deaths. Hospitalist. 2020; [Accessed June 1, 2020, https://www.the- hospitalist.org/hospitalist/article/220457/coronavirusupdates/comorbidities-rule-new-yorks-covid-19-deaths].

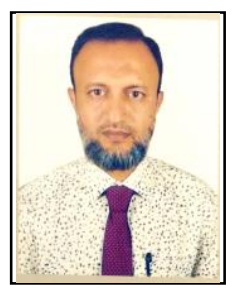

M M Bodiuzzaman was born in Gopalgonj, a district of Bangladesh on $3^{\text {rd }}$ October, 1972. He was graduated (MBBS) from M A G Osmani Medical College, Bangladesh on 1997 and did his post graduation (FCPS) in Internal Medicine from Bangladesh College of Physicians and Surgeons in 2012.

$\mathrm{He}$ is currently working in Faridpur Medical College as an Assistant Professor of Medicine. Prior to his recent posting he was working as Junior Consultant of Medicine in General Hospital, Faridpur, Bangladesh. He published a number of scientific papers in preferred Journals. His major works include: (i) Prevalence of modifiable risk factors among stroke patients in a tertiary care hospital, published in $\mathrm{J}$ Medicine 2009 January; 10(1):18-21. (ii) Use of oral valacyclovir instead of IV acyclovir in treatment of Herpes simplex encephalitis in resources poor country- A review article in Faridpur Med. Coll. J. 2019;14(1):4143.

Dr Zaman is a member of Bangladesh College of Physicians and Surgeons a renowned Medical Postgraduate Institute in Bangladesh.

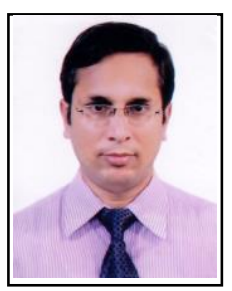

Mohammad Iqbal Hossain was born in Faridpur, a district of Bangladesh on $16^{\text {th }}$ of February 1978. He was graduated (MBBS) from Dhaka Medical College, Bangladesh on 2002 and did his post graduation (FCPS) in internal medicine from Bangladesh College of Physicians and Surgeons in 2011.

He is currently working in Faridpur Medical College as an Assistant Professor of Medicine. Prior to his recent posting he was working as Junior Consultant of Medicine in Upazila Health Complex of Kalukhale,

Rajbari, Bangladesh. He published a number of papers in preferred Journals. His major works include:

1. A Study of Hospital Outcome of Stroke Patients Admitted in A Tertiary Level Hospital published in 2019 July;6(2):43-46.

2. Study of Comorbidities among Stroke Patients Admitted in A Tertiary Level Hospital published in KYAMC Journal 2019;10(3):152-55.

3. A Study of Hospital Outcome of Liver Cirrhosis Patients Admitted in A Tertiary Level Hospital published in DCIMCJ 2020 January;7(1):52-56.

Dr. Hossain is a life member of Bangladesh College of Physicians and Surgeons, a renowned postgraduate institute in Bangladesh. 